\title{
Propiedades psicométricas de la Escala de Satisfacción con la Uida [SWLS] de Diener en población de michoacán (méxico)
}

\section{Psychometric Properties of Diener's Scale of Satisfaction with Life [SULS] in a Mexican Population \\ Propriedades Psicométricas da Escala de Satisfação com a Uida [SULS] de Diener na população de Michoacán [méxico]}

\author{
Ferran Padrós Blázquez, Claudia Yunuén Gutiérrez Hernández, \\ Mayra Areli Medina Calvillo* \\ Universidad Michoacana de San Nicolás de Hidalgo, México
}

Doi: dx.doi.org/10.12804/ap133.02.2015.04

\section{Resumen}

En la presente investigación se estudiaron las propiedades psicométricas de la Escala de Satisfacción con la Vida de Diener en población de Michoacán (México). Se aplicó la escala a dos grupos de participantes sanos ( $N=477$ en población estudiantil y 301 en población general). Los resultados sugieren que la escala se ajusta de forma excelente a un modelo unifactorial como en la versión original; además, se observó una elevada consistencia interna y una alta confiabilidad test-retest. También se evaluaron las posibles relaciones entre la escala de satisfacción con la vida y escalas de bienestar psicológico, depresión clínica y niveles de afecto positivo y negativo. Los datos empíricos indican que la escala de satisfacción con la vida presenta una validez y confiabilidad satisfactorias. Así mismo, se ofrecen de datos sobre la media y la desviación estándar en población de Michoacán (México).
Palabras clave: satisfacción con la vida; bienestar subjetivo; confiabilidad; validez; felicidad.

\section{Abstract}

The goal of our study is to establish the psychometric properties of Diener's Scale of Satisfaction with Life in a Mexican population. To perform our study we applied Diener's Scale of Satisfaction with Life to healthy volunteers from two samples (students, $N=477$ and general population, $N=301$ ). Our results suggest that the scale has an excellent adjustment to a unifactorial model, as the original one. The scale also has high internal consistency, and high test-retest reliability. We also tested the possible relationships between Diener's scale and psychological well-being, clinical depression, and positive and negative affect. Empirical data indicate that Diener's Scale of Satisfaction with Life presents satisfactory validity and reliability. In addition we also

* La correspondencia relacionada con este artículo debe ser dirigida a Ferran Padrós Blázquez, Facultad de Psicología, Universidad Michoacana de San Nicolás de Hidalgo, México. Correo electrónico: fpadros@uoc.edu

Para citar este artículo: Padrós, F., Gutiérrez, C. Y. \& Medina, M. A. (2015). Propiedades psicométricas de la Escala de Satisfacción con la Vida (SWLS) de Diener en población de Michoacán (México). Avances en Psicología Latinoamericana, 33(2), 221-230. doi: dx.doi.org/10.12804/ap133.02.2015.04 
present data on the mean score and standard deviation of Diener's scale in the Mexican population.

Key words: satisfaction with life; subjective well-being; reliability; validity; happiness.

\section{Resumo}

$\mathrm{Na}$ presente pesquisa estudaram-se as propriedades psicométricas da satisfação com a vida de Diener na população de Michoacán (México). Aplicamos a escala a dois grupos de participantes saudáveis ( $N=477$ de população estudantil e 301 de população geral); nossos resultados sugerem que a escala ajusta-se de forma excelente a um modelo unifatorial como na versão original; observou-se, alem disso, uma elevada consistência interna e uma alta confiabilidade teste-reteste. Também avaliamos as possíveis relações entre a escala de satisfação com a vida e escalas de bem-estar psicológico, depressão clínica e níveis de afeto positivo e negativo. Os dados empíricos indicam que a escala de satisfação com a vida apresenta uma validez e confiabilidade satisfatórias. Igualmente se oferecem dados sobre a média e desvio padrão na população de Michoacán (México). Palavras chave: satisfação com a vida; bem-estar subjetivo; confiabilidade; validez; felicidade.

Tradicionalmente, la psicología había centrado su interés en el estudio de la enfermedad y los trastornos mentales, y se había enfocado en el alivio de los estados negativos y relacionados con el malestar. Sin embargo, desde 1948, la Organización Mundial de la salud (OMS, 1948) concibió la salud como un estado completo de bienestar físico, psicológico y social, y dejó de ser considerada sinónimo de ausencia de enfermedad o minusvalía. Pero solo hasta finales del siglo XX el interés por conceptos como bienestar psicológico o bienestar subjetivo aumentó considerablemente. Debe señalarse que estos conceptos son el objeto central de la joven disciplina conocida como psicología positiva, cuyo objeto es el estudio científico las fuerzas y las virtudes humanas naturales; es una disciplina que se pregunta cuál es la naturaleza de la eficacia del funcionamiento del ser humano y centra la atención en los potenciales, motivos y capacidades de este (Sheldon \& King, 2001).

El principal objeto de estudio de la psicología positiva es el antiguo concepto de la felicidad, y en ella predominan dos acepciones que a su vez coinciden con antiguas corrientes filosóficas: la eudaimónica y la hedónica. El eudaimonismo, tradición iniciada por Aristóteles, está reflejado en el concepto de bienestar psicológico. Desde esta perspectiva se defiende que la opinión directa de los sujetos sobre si uno es feliz o no, no son representativos de lo que sería la "auténtica felicidad", en términos de Seligman (2003). El funcionamiento óptimo en diversas áreas es lo que define el grado de "felicidad" de la persona, aunque debe comentarse que se ha propuesto más de un modelo (Ryff, 1989; Ryan \& Deci, 2001).

Por otro, lado, la perspectiva hedónica tiene continuidad a través del concepto de bienestar subjetivo (Diener, 1984), el cual se ha definido como un constructo constituido por un componente cognitivo y dos afectivos. El nivel de "felicidad" de una persona, desde la perspectiva del bienestar subjetivo, supone preguntarle directamente a esta qué tan satisfecha con la vida está (el componente cognitivo) y en qué grado ha experimentado una serie de estados afectivos durante un periodo determinado, que posteriormente se agrupan en niveles de afecto positivo y negativo (los dos componentes afectivos).

Los dos tipos de afecto se conceptualizaron como dos dimensiones independientes a partir del trabajo de Bradburn (1969), ya que halló, mediante análisis psicométricos de un instrumento de medida, que surgían dos factores (en el análisis factorial) y que estos se relacionaban de modo distinto con otras variables, lo cual ha sido observado en estudios posteriores (Diener, 2009). Sin embargo, todavía existe un debate teórico-empírico respecto a si el afecto positivo y negativo es una sola 
dimensión bipolar o dos dimensiones unipolares independientes, puede verse una revisión en $\mathrm{Pa}$ drós-Blázquez, Navarro-Contreras y Soriano-Más (2012). El componente cognitivo se concibió como satisfacción con la vida (Andrews \& Withey, 1976; Diener, 1984; Diener, Suh, Lucas \& Smith, 1999), el cual se definió como "el grado en que una persona evalúa la calidad global de su vida en conjunto de forma positiva. En otras palabras, cuánto le gusta a una persona la vida que lleva" (Veenhoven, 1994, p. 90). La satisfacción con la vida es un una evaluación global sobre la calidad de vida que realiza una persona de acuerdo con sus criterios escogidos (Shin \& Johnson, 1978). La valoración sobre la satisfacción con la vida se puede evaluar como la suma de la satisfacción en dominios específicos (salud, familia, trabajo, amistades, tiempo libre, etc.) o, en general, mediante instrumentos relativamente extensos como el propuesto por Alfonso, Allison, Rader y Gorman (1996) de 50 reactivos, o mediante una o pocas preguntas, como es la escala que en el presente estudio nos ocupa.

Una posible utilidad de la evaluación de la satisfacción de vida es que puede considerarse una evaluación de calidad de vida; pero debe comentarse que son varias las acepciones de calidad de vida - en ocasiones incluyen aspectos objetivos en la evaluación-, pero en cualquier concepción de calidad de vida debe evaluarse de una forma $u$ otra la satisfacción con la vida (Moons, Budts \& De Geest, 2006).

Finalmente, debe destacarse una de las principales utilidades de la Escala de Satisfacción con la Vida, que es la de disponer de un instrumento que permite ser usado para tratar de ir dilucidando y promocionando las diferentes vías para acceder al bienestar, como ya se ha iniciado en Latinoamérica (Castro-Solano, 2011).

El objetivo de esta investigación fue valorar si las propiedades psicométricas de la Escala de Satisfacción con la Vida de Diener son adecuadas para población del centro de México.

\section{Método}

\section{Participantes}

En este estudio se utilizaron dos grupos de participantes voluntarios: el grupo A, el cual estuvo formado por 477 alumnos de la Facultad de Psicología de la Universidad Michoacana de San Nicolás de Hidalgo (UMSNH), de los cuales 245 participaron en el retest, y el grupo B, que se extrajo de la población general compuesto por 301 participantes. La media de edad del grupo A fue de $19.00(D T=2.18)$. Hasta un $78.62 \%$ de participantes fueron mujeres, y prácticamente la totalidad eran solteros (99.6\%). El grupo B estuvo constituido por un $51.49 \%$ de mujeres y un $48.5 \%$ de varones de la población general. El promedio de edad fue $29.03(D T=10.96)$ y la media de años de escolaridad resultó de $13.59(D T=3.93)$. Respecto al estado civil, un $68.4 \%$ eran solteros, un $29.5 \%$ vivía en pareja, un $1.5 \%$ manifestó estar divorciado o separado y $.8 \%$ eran viudos.

\section{Instrumentos}

Escala de Satisfacción con la Vida de Diener (Diener, Emmons, Larsen \& Griffin, 1985), que consta de cinco ítems que evalúan la satisfacción con la vida a través del juicio global que hacen las personas sobre esta. Para el presente trabajo se utilizó la traducción al castellano de Atienza, Pons, Balaguer y García-Merita (2000), la cual presenta los reactivos de la siguiente manera: (a) "En la mayoría de los aspectos mi vida es como quiero que sea", (b) "Hasta ahora he conseguido de la vida las cosas que considero importantes", (c) "Estoy satisfecho con mi vida", (d) "Si pudiera vivir mi vida otra vez, la repetiría tal y como ha sido" y (e) "Las circunstancias de mi vida son buenas". En esta versión se llevó a cabo una reducción en las opciones de respuesta del instrumento (en la versión original es de 7), por lo que los valores oscilan entre 1 y 5 , donde 1 es "totalmente en desacuerdo", 
y 5 es "totalmente de acuerdo". La puntuación total va de 5 (baja satisfacción) a 25 (alta satisfacción).

Este instrumento se ha utilizado en gran cantidad de estudios y ha mostrado obtener muy buenas propiedades psicométricas. Es una escala de un solo factor, y ha mostrado alta consistencia interna con valores de alfa de Cronbach, que oscilan entre .89 y.79. Se han obtenido correlaciones negativas de la escala con el afecto negativo, ansiedad y depresión, y por otro lado correlaciones positivas con otras medidas de bienestar (Diener et al., 1985).

Escalas de Afecto Positivo y Afecto Negativo (PANAS) de Watson, Clark y Tellegen (1988). Se utilizó la versión corta de las escalas adaptada a población mexicana por Robles y Páez (2003). Es un instrumento autoaplicable que consta de dos apartados de 20 reactivos cada uno, 10 de los cuales miden el afecto positivo, y 10, el afecto negativo. Se evalúa la presencia de los afectos referido a diferentes periodos (que oscilan desde en el momento en que se contesta hasta generalmente). En la presente investigación se utilizó la referida a generalmente. Los reactivos están formados por palabras que describen diferentes emociones y sentimientos y se contestan indicando con un número el grado en que es experimentado en un rango de 1 (muy poco o nada) a 5 (extremadamente).

Las escalas PANAS en población mexicana (Robles \& Páez, 2003) poseen valores de consistencia interna entre $\alpha=.84$ (afecto positivo) y $\alpha=$ .87 (afecto negativo); la correlación entre los dos afectos (positivo y negativo) es baja, entre $r=-.12$ y $r=-.23$. El instrumento cuenta con valores de confiabilidad test-retest entre $r=.71 \mathrm{y} r=.68$, lo cual sugiere que pueden emplearse para evaluar el afecto como un rasgo de personalidad estable en el tiempo y como estado.

\footnotetext{
Escala Multidimensional para la Medición del Bienestar Subjetivo (EMMBSAR), de Anguas Plata y Reyes Lagunes (1997). Instrumento que consta de 2 escalas (la Escala de Experien-
}

cia Emocional, la cual mide frecuencia e intensidad emocional, y la Escala de Evaluación Afectivo-Cognitiva de la Vida) y un pequeño cuestionario demográfico. Para el presente estudio se utilizó únicamente la Escala de Evaluación Afectivo-Cognitiva de la vida, que se compone de 6 factores (interacción total recibida, logros personales, religión, calidad de vida, clima familiar y logros nacionales), se conforma por 50 preguntas de 7 opciones de respuesta presentadas en un formato tipo Likert, donde las opciones están representadas por 7 cuadrados, ordenados de mayor a menor, de izquierda a derecha. El sujeto que responde debe marcar el cuadrado que mejor representa la cantidad de satisfacción. Posee elevada consistencia interna de $\alpha=.95$.

Inventario de Depresión de Beck (BDI). Este instrumento se diseñó para evaluar la intensidad de la sintomatología depresiva presente en un individuo. Se utilizó la versión mexicana adaptada por Jurado, Villegas, Méndez, Rodríguez, Loperena y Varela (1998). El instrumento es autoaplicable y consta de 21 reactivos, cada cual con 4 afirmaciones que describen diferentes categorías de tipo sintomático y conductual. El primer enunciado tiene un valor de 0 , que indica la ausencia del síntoma; el segundo, de 1 punto; el tercero, de 2 puntos, y el cuarto, de 3 puntos. El BDI presenta una consistencia interna de $\alpha=.87$ en población general, y cuenta con 3 factores. Las normas de calificación propuestas son: depresión mínima (de 0 a 9 puntos), depresión leve (de 10 a 16 puntos), depresión moderada (de 17 a 29 puntos) y depresión severa (de 30 a 63).

\section{Procedimiento}

En este estudio se decidió utilizar la versión original de la escala traducida al castellano por Atienza et al. (2000). Esto debido a que 4 psicólogos mexicanos con experiencia en el ámbito de la psicometría y con conocimientos sobre el bienestar 
subjetivo revisaron el instrumento y no consideraron pertinente la modificación de ningún reactivo.

La recolección de todos los datos de los grupos del presente trabajo se realizó después de que el proyecto fuera aprobado por el Comité de Ética de la Facultad de Psicología de la UMSNH. Todos los participantes firmaron un formato de consentimiento informado donde señalaban que aceptaban participar voluntariamente en la investigación.

Los datos del grupo de estudiantes se obtuvieron en dos etapas. En la primera fase se solicitó la participación voluntaria y anónima a los alumnos de Psicología de la Facultad de Psicología de la UMSNH, y se les administró un protocolo que contenía los siguientes instrumentos: la Escala de Satisfacción con la Vida, el Inventario de Depresión de Beck, la Escala Multidimensional para la Medición del Bienestar Subjetivo y las Escalas de Afecto Positivo y Afecto Negativo. En la segunda etapa se asistió a los mismos salones (dos meses de diferencia) y se les administró la Escala de Satisfacción con la Vida, con la finalidad de estudiar la confiabilidad test-retest. Los participantes se identificaron posteriormente, a través de un código que permitía emparejar de forma anónima los cuestionarios que habían sido respondidos por la misma persona.

Los datos del grupo B se recolectaron, a través de la técnica bola de nieve (Vogt, 1999), la cual consiste en que el investigador solicita la participación anónima y voluntaria a personas de la población general que pueden ser conocidos o personas que encuentra en la vía pública. Además, a las mismas personas que responden, se les pide a su vez que soliciten la participación de otras y así sucesivamente.

\section{Resultados}

Todos los análisis estadísticos se llevaron a cabo por medio del programa SPSS 17.0 para Windows con la excepción del el análisis factorial confirmatorio, que se efectuó con el programa de ecuaciones estructurales EQS (Bentler, 2005).

\section{Estructura interna}

Se realizó un análisis factorial confirmatorio con el grupo A y otro con el grupo B. Los parámetros del modelo se estimaron siguiendo el modelo de Máxima Verosimilitud. Los 5 ítems de la escala se agruparon en una sola variable latente, puesto que los datos basados en cuestionaros tipo Likert pueden aparecer en distribuciones que no se ajusten a la ley normal, y se utilizó el método de distribución ML-Robusto. Se emplearon los siguientes indicadores de bondad de ajuste: $\mathrm{S}-\mathrm{B} \chi^{2}=\chi^{2}$ escalado de Satorra-Bentler dividido por grados de libertad (valores $\leq 5.0$ y valores de $\mathrm{S}-\mathrm{B} \chi^{2}$ no significativos indican buen ajuste del modelo); GFI = LISREL goodness of fit index; $\mathrm{CFI}=$ comparative fit index (valores $\geq .90$ señalan buen ajuste); $\mathrm{SRMR}=$ standardized root mean square residual (valores $\leq .08$ indican buen ajuste); RMSEA = root mean square error of approximation (valores $\leq .06$ indican buen ajuste). Los resultados obtenidos sugieren una bondad de ajuste adecuada para el modelo de un solo factor (tabla 1).

Tabla 1

Índices de bondad de ajuste para el modelo de un factor (en los grupos $A$ y $B$ )

\begin{tabular}{cccccccc}
\hline Grupo & $\chi^{2}(g l)$ & $\chi^{2} / g l$ & $p$ & GFI & CFI & SRMR & RMSEA \\
\hline A & $\begin{array}{c}6.247 \\
(5)\end{array}$ & 1.2494 & .28292 & .994 & .998 & .017 & .024 \\
& & & & & & \\
B & $\begin{array}{c}19.758 \\
(5)\end{array}$ & 3.9516 & .07392 & .979 & .980 & .031 & .056 \\
& & & & & & &
\end{tabular}

Nota: índices corregidos (método robusto). $\mathrm{S}-\mathrm{B} \chi^{2}=\chi^{2}$ escalado de Satorra-Bentler; GFI $=$ LISREL goodness of fit index; $\mathrm{CFI}=$ comparative fit index; $\mathrm{SRMR}=$ standardized root mean square residual; RMSEA = root mean square error of approximation .

\section{Consistencia interna y estudio sobre la idoneidad de los reactivos}

El valor de alfa de Cronbach del total de la escala resultó de $\alpha=.832$ (en el grupo B de la población general). 
La puntuación media para cada ítem osciló entre 3.26 (ítem 4) y 3.87 (ítem 3), y los valores de las desviaciones estándar para cada ítem estuvieron alrededor de 1. El rango de correlaciones de cada uno de los ítems con la puntuación total corregida (eliminado el propio ítem) fue elevada, estuvo por encima de .6 en los 5 reactivos. También se observó que al eliminar cualquiera de los ítems el valor del alfa de Cronbach de la escala total disminuía considerablemente (tabla 2).

Tabla 2

Descriptivos de cada ítem (extraídos del grupo B)

\begin{tabular}{ccccc}
\hline $\begin{array}{c}\text { Ítem } \\
\text { número }\end{array}$ & Media & $D T$ & $\begin{array}{c}r \text { ítem y } \\
\text { escala } \\
\text { (sin ítem) }\end{array}$ & $\begin{array}{c}\text { alfa } \\
\text { (sin ítem) }\end{array}$ \\
\hline 01 & 3.56 & 1.02 & .629 & .800 \\
02 & 3.76 & 1.08 & .658 & .792 \\
03 & 3.87 & 1.07 & .703 & .779 \\
04 & 3.26 & 1.30 & .577 & .823 \\
05 & 3.84 & 0.94 & .628 & .802 \\
\hline
\end{tabular}

\section{Confiabilidad test-retest y relación con otras} variables

La correlación test-retest observada de la escala fue de $r=.575(N=335, p<.001)$, en un periodo de 2 meses aproximadamente. Se realizaron correlaciones de Pearson entre la Escala de Satisfacción con la Vida y las otras escalas a través de los datos extraídos del grupo de estudiantes.

La relación observada entre la Escala de Satisfacción con la Vida de Diener y la Escala de Evaluación Afectivo-Cognitiva de la EMMBSAR fue de $r$ $=.573$, escala con la que se observó la correlación más alta. Respecto a la escala que evalúa el afecto positivo fue de $r=.367$, con el afecto negativo fue de $r=-.255$ y con la escala de sintomatología depresiva fue de $r=-.324$ (todas las correlaciones con $p<.001)$.

\section{Datos en población de Michoacán (México)}

La media de la puntuación total de la Escala de Satisfacción con la Vida en el grupo de estudiantes fue de $19.15(D E=3.51)$ y la del grupo de la población general fue de $18.28(D E=4.21)$. En ambos grupos los valores mínimo y máximo fueron 5 y 25.

Se estudió si existían diferencias en los niveles de satisfacción con la vida en función del género, $\mathrm{y}$ se observó que la media reportada por las mujeres $(\bar{x}=18.45 ; D E=4.26)$ no fue significativamente diferente $\left(\mathrm{t}_{[299]}=.690 ; p=.491\right)$ a la observada en los hombres $(\bar{x}=18.11 ; D E=4.16)$. Así mismo, tampoco se observaron relaciones entre la satisfacción con la vida y la edad $(r=-.011 ; p=.850)$; pero sí se observó que a mayor número de años de escolarización, mayor nivel de satisfacción con la vida $(r=-.206 ; p<.001)$. Respecto al estado civil, se comparó la media obtenida por los solteros $(\bar{x}=$ $18.22 ; D E=4.12$ ) con la obtenida por las personas casadas o en unión libre $(\bar{x}=18.42 ; D E=4.52)$ y no resultó significativa $\left(\mathrm{t}_{[292]}=.365 ; p=.716\right)$.

\section{Discusión}

El objetivo principal del presente trabajo fue valorar si las propiedades psicométricas de la Escala de Satisfacción con la Vida son adecuadas para una población del centro de México. Respecto a la estructura factorial, los resultados del análisis factorial confirmatorio indican que el ajuste al modelo unidimensional es adecuado. Dichos resultados coinciden con los hallados en la versión original (Diener et al., 1985) y estudios posteriores realizados en población española (Atienza et al., 2000; Garrido-Muñoz de Arenillas, Fernández-Borrero, Villalba-Ruiz, Pérez-Moreno \& Fernández-Bellido, 2010; Vázquez, Duque \& Hervás, 2013), peruana (Martínez-Uribe, 2004), brasileña (Albuquerque, Sousa \& Martins, 2010), holandesa (Arrindell, Heesink \& Feij, 1999; Arrindell, Meeuwesen \& Huyse, 1991), alemana (Glaesmer, Grande, Brae- 
hler \& Roth, 2011) y malaya (Swami \& Chamorro-Premuzic, 2009). El valor observado del alfa de Cronbach del total de la escala es elevado (sobre todo teniendo en cuenta que solo cuenta con 5 reactivos); además, está dentro del rango señalado por la versión original de Diener et al. (1985) y coincide con lo reportado en otros trabajos (Pavot \& Diener 1993). Sin embargo, es inferior al hallado en el estudio de Eid y Diener (2004), que hallaron valores por encima de .90 .

Respecto a los reactivos, se observa que todos obtienen propiedades psicométricas adecuadas. En los cinco reactivos se observan puntuaciones por encima de 3 y por debajo de 4 (valores ligeramente por encima del valor medio las posibilidades que ofrece la escala, que sería 2.5), lo cual coincide con lo reportado en otros estudios (Pavot \& Diener, 1993; Atienza et al., 2000).

Los valores obtenidos en el test-retest sobre la escala de satisfacción con la vida sugieren una estabilidad temporal congruente con la conceptualización teórica y con los hallados en los estudios previos (Diener et al., 1985; Lucas, Diener \& Suh, 1996; Pavot \& Diener, 1993).

En cuanto a la relación observada entre la satisfacción con la vida con el afecto positivo y negativo, es similar a lo hallado en otros estudios (Lucas et al., 1996; Pavot, Diener, Randall Colvin \& Sandvik, 1991; Pavot \& Diener 1993). La relación obtenida con la presencia de sintomatología depresiva también es la esperada y la observada en estudios previos (Diener et al., 1985). Debe destacarse que se halló una correlación positiva y elevada con la Evaluación Afectivo-Cognitiva de la vida de la Escala de Medición del Bienestar Subjetivo. Quisiéramos señalar que no lo suficientemente elevada como para inferir que se trata de una escala breve que mide el mismo constructo. Nótese que realizar la medición por dominios tiene la ventaja de que al evaluado se le hace concretar en la autoevaluación en diferentes dominios que frecuentemente son relevantes para la mayoría de personas y que al responder de forma genérica puede no tener presente. Sin embargo, no para todos los seres humanos los ámbitos relevantes son los mismos y, sobre todo, pueden tener un peso diferente en cuanto a la relevancia que consideran cada uno de los aspectos (Diener et al., 1985). De modo que dichas diferencias individuales pueden subsanarse con una evaluación más genérica como la Escala de Satisfacción con la Vida de Diener. Pero debe comentarse que probablemente sea más sensible a otros sesgos como el estado anímico en el que se conteste la escala (Schwarz \& Clore, 1983; Schwarz \& Strack, 1991) o la deseabilidad social (Carstensen \& Cone, 1983).

En los resultados referentes a los niveles de satisfacción con las variables sociodemográficas se observa ausencia de relación respecto a la edad y el sexo, lo cual coincide con lo reportado en otros estudios (Arrindell et al., 1991; Pavot \& Diener, 1993; Glaesmer et al., 2011; Swami \& Chamorro-Premuzic, 2009; Vázquez et al., Duque \& Hervás, 2013). Sin embargo en el ámbito latinoamericano, se han reportado mayores niveles de satisfacción con la vida en adultos tardíos respecto a adolescentes (Martínez-Uribe, 2004). Por otro lado, los resultados obtenidos referidos a los años de escolarización también coinciden con lo hallado en estudios previos (Pavot \& Diener, 1993; Vázquez et al., 2013).

Respecto a la generalización de los valores obtenidos a la población mexicana, primero, debe señalarse que los grupos solo se han obtenido de Michoacán, que es solo un estado de México; por ello, deberían capturarse muestras de otros estados de la República mexicana. Además, también debe considerarse un posible sesgo de respuesta derivado del muestreo utilizado para obtener los datos de la población general, ya que es posible que aquellos participantes con menores niveles de satisfacción con la vida rehúsen contestar voluntariamente a este tipo de escalas.

Finalmente, quisiéramos subrayar la relevancia y utilidad de la Escala de Satisfacción con la Vida de Diener, debido a la facilidad y rapidez de apli- 
cación ha sido propuesto como índice nacional en Estados Unidos (Diener, 2000). Teniendo en cuenta las buenas características psicométricas observadas en las distintas culturas, podría ser considerado un índice transnacional para realizar estudios comparativos entre distintas culturas, como el realizado por Diener y Eunkook (2003).

\section{Referencias}

Albuquerque, F. J. B., Sousa, F. M. \& Martins, C. R. (2010). Validação das escalas de satisfação com a vida e afetos para idosos rurais. Psico, 41(1), 85-92. Recuperado de http://revistaseletronicas.pucrs.br/ojs/index.php/revistapsico/ article/view/5110

Alfonso, V. C., Allison, D. B., Rader, D. E. \& Gorman, B. S. (1996). The extended satisfaction with life scale: Development and psychometric properties. Social Indicators Research, 38, 275-301. Recuperado de http://link.springer. com/article/10.1007\%2FBF00292049\#page-2

Andrews, F. M. \& Withey, S. B. (1976). Social indicators of well-being: American's Perceptions of life quality. New York: Plenum Press.

Anguas, P. \& Reyes, L. (1997). El significado del bienestar, su valoración en México. Tesis de maestría no publicada, Facultad de Psicología de la Universidad Autónoma de México (UNAM).

Arrindell, W. A., Heesink, J. \& Feij, J. A. (1999). The Satisfaction with Life Scale SWLS): appraisal with 1700 healthy young adults in The Netherlands. Personality and Individual Differences, 26, 815-826. Recuperado de http://www.sciencedirect.com/science/article/pii/ S0191886998001809

Arrindell, W. A., Meeuwesen, L. \& Huyse, F. J. (1991). The Satisfaction with Life Scale (SWLS): Psychometric properties in a non-psychiatric medical outpatients sample. Personality and Individual Differences, 12(2), 117-123. Recuperado de http://www.sciencedirect.com/ science/article/pii/019188699190094R
Atienza, F., Pons, D., Balaguer, I. \& García-Merita, M (2000). Propiedades psicométricas de la escala de satisfacción con la vida en adolescentes. Psychotema, 12,314-319. Recuperado de http:// www.unioviedo.es/reunido/index.php/PST/article/view/7597

Bentler, P. M. (2005). EQS Structural equations program manual. Encino, CA: Multivariate Software.

Bradburn, N. M. (1969). The Structure of Psychological Well-being. Chicago: Aldine.

Carstensen, L. L. \& Cone, J. D. (1983). The influence of social desirability on the measurement of psychological well being in Elderly Persons. Journal of Gerontology, 38, 713-715. Recuperado de http://geronj.oxfordjournals.org/content $/ 38 / 6 / 713$.short

Castro-Solano, A. (2011). Las rutas del acceso al bienestar: relaciones entre bienestar hedónico y eudaimónico. Un estudio en población argentina. Revista Iberoamericana de Diagnóstico y Evaluación Psicológica. 26(2), 117-139. Recuperado de http://www.aidep.org/03_ridep/2_volumen31.html

Diener, E. (1984). Subjective Well-being. Psychological Bulletin, 95(3), 542-575. Recuperado de http://internal.psychology.illinois.edu/ ediener/ review.html

Diener, E. (2000). Subjective well-being: The science of happiness and a proposal for a national index. American Psychologist, 55, 34-43. Recuperado de http://www.ncbi.nlm.nih.gov/ pubmed/11392863

Diener, E. (2009). Introduction-The science of well-being: Reviews and theoretical articles by Ed Diener. In E. Diener (Ed.), The science of well-being: The collected works of Ed Diener (pp. 1-10). New York: Springer.

Diener, E., Emmons, R. A., Larsen, R. J. \& Griffin, S. (1985). The satisfaction with life scale. Journal of Personality Assessment, 49, 71-75. Recuperado de http://internal.psychology.illinois. edu/ ediener/review.html 
Diener, E. \& Eunkook, M.S. (2003). National differences in subjective well-being. En D. Kahneman, E. Diener y N. Schwartz (Eds.), Well-being: The foundations of hedonic psychology (pp. 434-450). New York: Russell Sage Foundation.

Diener, E., Suh, E., Lucas, R. \& Smith, H. (1999). Subjetive well-being: three decades of progress. Psychological Bulletin, 125(2), 276-302. Recuperado de http://internal.psychology.illinois. edu/ ediener/review.html

Eid, M. \& Diener, E. (2004). Global judgments of subjective well-being: Situational variability and long-term stability. Social Indicators Research, 65, 245-277. Recuperado de http://link. springer.com/article/10.1023\%2FB\%3ASOCI.0000003801.89195.bc\#page-1

Garrido Muñoz de Arenillas, M. R., Fernández Borrero, M. A., Villalba Ruiz, E. B., Pérez Moreno, P. J. \& Fernández Bellido, M. (2010). Evidencias de validez de la adaptación al español de la escala de satisfacción con la vida (SWLS) en una muestra de estudiantes universitarios. Metodología de Encuestas (12), 45-62. Recuperado de http://casus.usal.es/pkp/index.php/ MdE/article/view/1002

Glaesmer, H., Grande, G., Braehler, E. \& Roth, M. (2011). The German version of the satisfaction with Life Scale (SWLS): psychometric properties, validity, and population-based norms. European Journal of Psychological Assessment, 27, 127-132. Recuperado de http://psycnet.apa. org/journals/jpa/27/2/127/

Jurado, S., Villegas, M. E., Méndez, L. Rodríguez, F. Loperena, V. \& Varela, R. (1998). La estandarización del Inventario de Depresión de Beck para los residentes de la ciudad de México. Salud Mental, 21, 26-31. Recuperado de http:// www.imbiomed.com.mx/1/1/articulos.php?method=showDetail\&id_articulo=11318\&id_seccion $=3 \&$ id_ejemplar $=1169$ \&id_revista $=1$

Lucas, R. E., Diener, E. \& Suh, E. (1996). Discriminant Validity of Well-Being Measures. Journal of Personality and Social Psychology, 71(3),
616-628. Recuperado de http://www.ncbi.nlm. nih.gov/pubmed/8831165

Martínez-Uribe, P. (2004). Perspectiva temporal futura y satisfacción con la vida a lo largo del ciclo vital. Revista de Psicología, 22(2), 215-252. Recuperado de http://dialnet.unirioja.es/servlet/ articulo? codigo $=1112231$

Moons, P., Budts, W. \& De Geest, S. (2006). Critique on the conceptualisation of quality of life: A review and evaluation of different conceptual approaches. International Journal of Nursering Studies, 43(7), 891-901. Recuperado de http://www.sciencedirect.com/science/article/ pii/S0020748906001088

Organización Mundial de la Salud. (1948). World Health Organization constitution. Geneve: Author.

Pavot, W. \& Diener, E. (1993). Review of the Satisfaction with Life Scale. Psychological Assessment, 5(2), 164-172. Recuperado de http://internal. psychology.illinois.edu/ ediener/review.html

Pavot, W., Diener, E., Randall Colvin, C. \& Sandvik, E. (1991). Further Validation of the Safisfaction with Life Scale: Evidence for the cross-method convergence of well-being measures. Journal of Personality Assessment, 57(1), 149-161. Recuperado de http://internal.psychology.illinois. edu/ ediener/SWLS.html

Padrós-Blázquez, F., Contreras-Navarro, G. \& Soriano-Más, C. (2012). Afecto positivo y negativo: ¿una dimensión bipolar o dos dimensiones unipolares independientes? Interdisciplinaria y Ciencias Afines, 29(1), 1-14. Recuperado de http://www.scielo.org.ar/scielo.php?pi$\mathrm{d}=\mathrm{S} 1668-70272012000100009 \&$ script $=$ sci arttext

Robles, R. \& Páez, F. (2003). Estudio sobre la traducción al español y las propiedades psicométricas de las Escalas de Afecto Positivo y Negativo (PANAS). Salud Mental, 26, 69-75. Recuperado de http://www.researchgate.net/ publication/26475474_Estudio_sobre_la_traduccin_al_espaol_y las propiedades_psicom- 
tricas_de_las_escalas_de_afecto_positivo_y_ negativo_\%28panas\%29

Ryan, R.M. \& Deci, E.L. (2001). On happiness and human potentials: A review of research on hedonic and eudaimonic well-being. Annual Review of Psychology, 52, 141-166.

Ryff, C. D. (1989). Happiness is everything, or is it?: Explorations on the meaning of psychological well-being. Journal of Personality and Social Psychology, 57(6), 1069-1081. Recuperado de http://psycnet.apa.org/journals/psp/57/6/1069/

Schwarz, N. \& Clore, G. L. (1983). Mood, misattribution and judgement of well-being. Informative and directive functions of affective states. Journal of Personality and Social Psychology, 45, 513-523. Recuperado de http://psycnet. apa.org/index.cfm?fa $=$ search.displayRecord\&uid=1984-12290-001

Schwarz, N. \& Strack, F. (1991). Evaluating one's life: A judgement model of subjective well-being. In F. Strack, M. Argyle \& N. Schwarz (Eds.), Subjective Well-being: An interdisciplinary perspective (pp. 27-48). Oxford: Oxford University Press.

Seligman, M. E. P. (2003). La auténtica felicidad. Barcelona: Vergara.

Sheldon, K. M. \& King, L. (2001). Why positive psychology is necessary? American Psychologist, 56, 216-217. Recuperado de http://www.ncbi.nlm. nih.gov/pubmed/11315247

Fecha de recepción: 17 de mayo de 2013 Fecha de aceptación: 24 de noviembre de 2014
Shin, D. \& Johnson, D. (1978). Avowed happiness as an overall assessment of the quality of life. Social Indicators Research, 5, 475-492. Recuperado de http://link.springer.com/article/10.1007\%2FBF00352944

Swami, V. \& Chamorro-Premuzic, T. (2009). Psychometric evaluation of the Malay satisfaction with life scale. Social Indicators Research, 92, 25-33.

Vázquez, C., Duque, A. \& Hervás, G. (2013). The Satisfaction with Life Scale (SWLS): Adaptation and normative data in a Spanish representative national sample. Spanish Journal of Psychology. Recuperado de http://pendientedemigracion. ucm.es/info/psisalud/carmelo/publicaciones. html

Veenhoven, R. (1994). El estudio de la satisfacción con la vida. Intervención Psicosocial, 3, 87-116. Recuperado de http://repub.eur.nl/res/ $\mathrm{pub} / 16195 /$

Vogt, W. P. (1999). Dictionary of statistics and methodology: A nontechnical guide for the social sciences (2nd ed.). Thousand Oaks London: Sage.

Watson, D., Clark, L. A. \& Tellegen, A. (1988). Development and validation of brief measures of positive and negative affect: The PANAS scales. Journal of Personality and Social Psychology, 54, 1063-1070. Recuperado de http://www.ncbi. nlm.nih.gov/pubmed/3397865 